\title{
Battle of the sex steroids in the male skeleton: and the winner is...
}

\author{
Thomas J. Weber \\ Division of Endocrinology, Metabolism and Nutrition, Department of Medicine, Duke University Medical Center, Durham, North Carolina, USA.
}

\begin{abstract}
Male osteoporosis is a multifactorial disease, although it is often in part related to hypogonadism. While testosterone replacement therapy has been shown to improve bone mineral density, studies have also linked bone loss and higher fracture risk in men to low estrogen levels. In this issue of the $J C l$, Finkelstein and colleagues report the results of a clinical study in a cohort of healthy adult men aimed at further discerning the specific roles of androgen and estrogen deficiency in bone loss. The results of their study support previous findings that estrogen deficiency has a dramatic effect on bone homeostasis in men. Future studies to corroborate and expand on these findings have potential to influence the clinical management of male osteoporosis.
\end{abstract}

\section{Background}

Male osteoporosis is an important public health problem that affects approximately 2 million men in the US and 5.5 million men in the European Union. This condition is currently responsible for approximately 600,000 fractures and over $\$ 4$ billion in direct medical costs in the US (1). Although male osteoporosis is often heterogeneous in nature and typically results from more than one contributing factor, male hypogonadism commonly underlies the disease. Androgen deficiency has profound skeletal effects, resulting in reduced trabecular and cortical bone density (2). In keeping with this primary defect, testosterone replacement therapy in hypogonadal men does restore bone homeostasis and improves bone mineral density (3); however, studies have also suggested that lower baseline testosterone levels $(<200$ $\mathrm{ng} / \mathrm{dl}$ ) are clinically predictive of a bone mineral density response (4). Additionally, there is no substantial evidence that testosterone replacement reduces the risk of osteoporotic fracture.

Within the past two decades, clinical observations have provided fundamental insight into the nature of sex steroid regulation of male skeletal biology. Specifically, the identification of an inactivating mutation of estrogen receptor $\alpha$ in a man with severe and refractory osteoporosis (5) and the identification of treatable estrogen deficiency due to mutation of the CYP19A1 gene and defective aromatization confirmed the obligatory nature of estrogen in bone mass accrual and maintenance (6). Subsequent studies have corroborated these findings and have linked estradiol deficiency to more rapid rates of bone loss in older men $(7,8)$. Additionally, treatment with dihydrotestosterone, a nonaromatizable androgen that reduces estradiol levels, and aromatase inhibitors provoke substantial bone mineral density loss in men $(9,10)$. Furthermore, there is a stronger inverse association between estrogen level and fractures in older men than testosterone $(11,12)$. Although these studies are compelling, others have found that androgen deficiency predicts fracture risk independent of estrogen status in men (13). It is clear that androgen receptor signaling contributes to the maintenance of trabecular and cortical

Related Article: p. 1114

Conflict of interest: The author has declared that no conflict of interest exists

Reference information: J Clin Invest. 2016;126(3):829-832. doi:10.1172/JCI85006.

bone, as androgen receptor $\mathrm{KO}$ mice have reduced bone volume (14). Furthermore, men with complete androgen sensitivity have diminished bone density in spite of adequate estrogen levels, supporting an obligatory role for androgens in the accrual and/or maintenance of bone density in men $(15,16)$. Together, these findings question the relative independent contributions of estrogen and testosterone to male skeletal metabolism.

\section{Current and previous findings}

In this issue, Finkelstein and colleagues attempted to further dissect the relative contributions of estrogen and testosterone to skeletal homeostasis (17). Specifically, young men were rendered either testosterone deficient (cohort 1) or both testosterone and estrogen deficient (cohort 2) via a gonadotropin-releasing hormone (GnRH) agonist without or with aromatase inhibitor letrozole, respectively. Testosterone levels less than $200 \mathrm{ng} / \mathrm{dl}$ and estradiol levels less than $10 \mathrm{pg} / \mathrm{ml} \mathrm{dra-}$ matically increased bone resorption, as measured by serum C-telopeptide (CTX), in the presence of testosterone deficiency alone. Induction of more severe estradiol deficiency $(<2.8 \mathrm{pg} / \mathrm{ml})$ profoundly increased bone resorption, and this effect was independent of testosterone level or testosterone replacement dose. Procollagen type-1 amino-terminal propeptide (P1NP), a marker of bone formation, was elevated in men with very low estradiol levels $(<5 \mathrm{pg} / \mathrm{ml})$, though this marker did not consistently correlate with higher levels of estradiol and testosterone. Consistent with bone resorption results, bone mineral density loss was generally greater in estrogen-deficient men and independent of testosterone level. Finally, indices of microarchitectural deterioration, including cortical thickness and porosity, as measured by high-resolution peripheral quantitative computerized tomography (HRpQCT), were modest but consistent with an independent effect of estradiol 
deficiency, although corresponding data in the isolated testosterone-deficient cohort was not procured. Together, these data confirm a dominant effect of estradiol in regulating bone homeostasis in younger men.

The study by Finkelstein and colleagues (17) confirms and extends previous investigations that support a role for estrogens in bone homeostasis in men. However, this study may also further discern sex steroid thresholds that are concerning for untoward bone effects in men. A seminal study by Falahati-Nini and colleagues elegantly demonstrated a dominant effect of estradiol on bone resorption and formation over three weeks in older men who were both estrogen and testosterone deficient, though sex steroids were measured by a less sensitive and specific method (radioimmunoassay) than that used by Finkelstein et al. (liquid chromatographytandem mass spectroscopy [LCMS]) (17, 18). Sanyal and colleagues found similar results in men on an abbreviated threeweek protocol, albeit with an apparent larger increase in bone resorption with estradiol and testosterone deficiency as measured by serum CTX (19). However, in contrast to Finkelstein et al. (17), P1NP was also lower in the Sanyal et al. study, perhaps due to an inability to capture recoupling of bone formation to resorption over a short time frame (19). Leder and colleagues also confirmed an important effect of estradiol in younger men through a similar, albeit longer (12-week) investigation, though the magnitude and discrimination of effects between estradiol and testosterone deficiency were less pronounced (20). These differences could be related to the prior use of immunoassays and urinary bone resorption markers, which have greater intraindividual variation than serum measures (21). Perhaps most importantly, the study by Finkelstein and colleagues (17) extends previous observations and confirms a deleterious effect of estrogen deficiency on bone macroarchitecture (bone mineral density) and microarchitecture in a younger population who would theoretically be less prone to such a decrement.

While the findings of Finkelstein et al. are certainly intriguing in aggregate, they would confer maximal clinical significance if they also predicted an increased risk of fracture in men (17). As detailed above, there is prospectively acquired data that does associate higher fracture risk in older men with lower estrogen levels (11, 12). More importantly, there appears to be a fairly consistent threshold estrogen level $(\sim 16 \mathrm{pg} / \mathrm{ml})$, below which the risk of fracture appears to increase in an exponential manner. This level is somewhat higher than the $10 \mathrm{pg} / \mathrm{ml}$ level reported by Finkelstein et al. to confer increased skeletal catabolism in younger men (17). These discrepancies may potentially be reconciled by differences in estrogen assay type and sensitivity, as well as by differences in patient populations (young vs. old). Indeed, higher levels of sex hormone-binding globulin in older men likely reduce free and/or bioavailable estrogen levels, thereby possibly confounding the interpretation of total estradiol levels in an older population.

\section{Unresolved issues/future directions}

Although the data presented by Finkelstein and colleagues strongly suggest that estrogen, irrespective of sex, is the dominant sex steroid in male bone metabolism, there is evidence that other sex steroids and factors contribute to male skeletal health (17). For example, testosterone appears to be critical for periosteal bone expansion that occurs both during growth and with aging in older men, likely conferring a biomechanical advantage for men over women for many types of appendicular fracture $(22,23)$. Additionally, testosterone deficiency independently predicts fracture risk in some populations (13). Perhaps most convincingly, a recent study in mice supports skeletal site-specific effects of estrogens and androgens by showing that targeted deletion of the murine androgen receptor-encoding gene in osteoblast progenitors compromises trabecular but not cortical bone (24). Conversely, complementary studies in men confirm that serum estradiol is positively associated with cortical thickness and inversely associated with cortical porosity, with evidence of a threshold effect on structural bone parameters, as well (25-27). These data prompted Khosla and colleagues to recharacterize bone resorption data of Falahati-Nini et al. in a model that is consistent with estrogendependent HRpQCT changes in cortical bone observed by Finkelstein et al., sug- gesting that sex steroid regulation of bone may well be compartment specific $(17,18$, 28). This model postulates that androgens and estrogens in men primarily modulate cancellous and cortical bone, respectively, though observed anabolic effects on cortical bone by both sex steroids may well be ligand independent (29). As cortical bone compromises approximately $80 \%$ of the adult human skeleton, the proportionate effects of estrogen and androgen deficiency on bone turnover would be consistent with this hypothesis. Moreover, current evidence based on animal models of estrogen deficiency and longitudinal, prospective studies in humans supports a life-long pattern of trabecular bone loss and a higher estrogen threshold for this loss that is likely exacerbated by cell-specific senescence-related changes, in contrast to a lower estrogen threshold required for cortical bone loss (30). Regarding other potential factors in male osteoporosis, studies have shown that higher sex hormone-binding globulin levels are associated with a higher risk of fracture in men, perhaps due to a direct effect on bone or an indirect effect as the result of lowering free and/or bioavailable sex steroid levels $(12,31)$. In addition, an indirect contribution of androgen deficiency to fracture risk in men due to reduced muscle mass and increased fall risk is also plausible. Finally, it is important to mention the potential contribution of nongenomic and ligand-independent effects of both testosterone and estrogen on bone health, although existing in vivo animal data indicate that such factors do not generally compensate for the negative skeletal effects of frank sex-steroid deficiency (32).

Despite the compelling evidence that adequate estrogen levels are critical for normal bone health in men, much more work is needed before these findings can be translated into meaningful clinical care. Foremost, the ability to accurately measure serum estradiol is paramount to these efforts. Although improved, immunoassays for determining levels of sex steroids have continued challenges related to sensitivity, accuracy, and interference by other factors (33). As such, the use of LCMS, as was used by Finkelstein et al. (17), should be considered, given that this technique has become more widely and commercially available. Increased utilization of LCMS may ultimately reduce the 
cost of these assays and possibly facilitate the use of serum estradiol to help guide decisions on management (such as the frequency of bone mineral density testing) and treatment (bisphosphonates, etc.). Randomized, controlled clinical trials will be necessary to establish efficacy of both hormonal and nonhormonal antifracture therapies in men with both de novo and induced estrogen deficiency. Although testosterone replacement of hypogonadal men with presumed normal aromatization activity is a logical trial choice (with obvious attention to extraskeletal risk and benefit), intriguing pilot studies also suggest a potential bone benefit from selective estrogen receptor modulators in men with low estradiol levels $(34,35)$. Indeed, the results of the bone trial substudy of the testosterone trials are eagerly awaited, as demonstration of inadequate bone mineral density improvement in men who remain estrogen deficient despite normalization of androgen status would confirm and extend the findings of Finkelstein and colleagues and further establish estrogen signaling as a potential target for osteoporosis treatment in men $(17,36)$. Notwithstanding these observations, there is also evidence that serum levels of estrogen (and testosterone) may not provide additional utility in the prospective prediction of osteoporotic fractures, which is obviously integral to potential clinical utility of such measurements (37). Finally, it will be critical to determine the relative contribution and the interrelated nature of additional factors - such as IGF-1, 25-OH $\mathrm{D}$, frailty, and falls - that are known to influence bone health and fracture risk in men (32). In light of the aggregate data to date, it is more appropriate to use betterestablished clinical surrogates for fracture risk prediction in men - including femoral neck bone mineral density, prior fractures, and FRAX scores - until sex steroid measurements are truly ready for prime time in the clinic $(38,39)$. Until then, we will all eagerly anticipate and enjoy the further unraveling of this truly fascinating aspect of bone biology.

\section{Acknowledgments}

The author would like to thank Eric Orwoll, Kenneth Lyles, and L. Darryl Quarles for their insightful feedback on this commentary.
Address correspondence to: Thomas J. Weber, 303 Baker House, DUMC 3470, Durham, North Carolina 27710, USA. Phone: 919.668.1367; E-mail: thomas. weber2@duke.edu.

1. Watts NB. Osteoporosis in men. Endocr Pract. 2013;19(5):834-838.

2. Finkelstein JS, Klibanski A, Neer RM, Greenspan SL, Rosenthal DI, Crowley WF Jr. Osteoporosis in men with idiopathic hypogonadotropic hypogonadism. Ann Intern Med. 1987;106(3):354-361.

3. Wang C, et al. Long-term testosterone gel (AndroGel) treatment maintains beneficial effects on sexual function and mood, lean and fat mass, and bone mineral density in hypogonadal men. JClin Endocrinol Metab. 2004;89(5):2085-2098.

4. Snyder PJ, et al. Effect of testosterone treatment on body composition and muscle strength in men over 65 years of age. J Clin Endocrinol Metab. 1999;84(8):2647-2653.

5. Smith EP, et al. Estrogen resistance caused by a mutation in the estrogen-receptor gene in a man. N Engl J Med. 1994;331(16):1056-1061.

6. Bilezikian JP, Morishima A, Bell J, Grumbach MM. Increased bone mass as a result of estrogen therapy in a man with aromatase deficiency. NEngl J Med.1998;339(9):599-603.

7. Fink HA, et al. Association of testosterone and estradiol deficiency with osteoporosis and rapid bone loss in older men. J Clin Endocrinol Metab. 2006;91(10):3908-3915.

8. Gennari L, et al. Longitudinal association between sex hormone levels, bone loss, and bone turnover in elderly men. J Clin Endocrinol Metab. 2003;88(11):5327-5333.

9. Idan A, et al. Long-term effects of dihydrotestosterone treatment on prostate growth in healthy, middle-aged men without prostate disease: a randomized, placebo-controlled trial. Ann Intern Med. 2010;153(10):621-632.

10. Burnett-Bowie SA, Roupenian KC, Dere ME, Lee $\mathrm{H}$, Leder BZ. Effects of aromatase inhibition in hypogonadal older men: a randomized, double-blind, placebo-controlled trial. Clin Endocrinol (Oxf). 2009;70(1):116-123.

11. LeBlanc ES, et al. The effects of serum testosterone, estradiol, and sex hormone binding globulin levels on fracture risk in older men. J Clin Endocrinol Metab. 2009;94(9):3337-3346.

12. Mellstrom D, et al. Older men with low serum estradiol and high serum SHBG have an increased risk of fractures.J Bone Miner Res. 2008;23(10):1552-1560.

13. Meier C, et al. Endogenous sex hormones and incident fracture risk in older men: the Dubbo Osteoporosis Epidemiology Study. Arch Intern Med. 2008;168(1):47-54.

14. Venken K, et al. Relative impact of androgen and estrogen receptor activation in the effects of androgens on trabecular and cortical bone in growing male mice: a study in the androgen receptor knockout mouse model. JBone Miner Res. 2006;21(4):576-585.

15. Sobel V, Schwartz B, Zhu YS, Cordero JJ, Impera-
to-McGinley J. Bone mineral density in the complete androgen insensitivity and $5 \alpha$-reductase- 2 deficiency syndromes. J Clin Endocrinol Metab. 2006;91(8):3017-3023.

16. Hughes IA, Davies JD, Bunch TI, Pasterski V, Mastroyannopoulou K, MacDougall J. Androgen insensitivity syndrome. Lancet. 2012;380(9851):1419-1428.

17. Finkelstein JS, et al. Gonadal steroid-dependent effects on bone turnover and bone mineral density in men. J Clin Invest. 2016;126(3):1114-1125.

18. Falahati-Nini A, Riggs BL, Atkinson EJ, O'Fallon WM, Eastell R, Khosla S. Relative contributions of testosterone and estrogen in regulating bone resorption and formation in normal elderly men. J Clin Invest. 2000;106(12):1553-1560.

19. Sanyal A, et al. Regulation of bone turnover by sex steroids in men. JBone Miner Res. 2008;23(5):705-714.

20. Leder BZ, LeBlanc KM, Schoenfeld DA, Eastell R, Finkelstein JS. Differential effects of androgens and estrogens on bone turnover in normal men. J Clin Endocrinol Metab. 2003;88(1):204-210.

21. Vasikaran S, et al. Markers of bone turnover for the prediction of fracture risk and monitoring of osteoporosis treatment: a need for international reference standards. Osteoporos Int. 2011;22(2):391-420

22. Seeman E. Osteoporosis in men. Osteoporos Int 1999;9(suppl 2):S97-S110.

23. Cummings SR, Melton LJ. Epidemiology and outcomes of osteoporotic fractures. Lancet. 2002;359(9319):1761-1767.

24. Ucer S, et al. The effects of androgens on murine cortical bone do not require AR or ER $\alpha$ signaling in osteoblasts and osteoclasts. J Bone Miner Res. 2015;30(7):1138-1149.

25. Vandenput L, et al. Serum estradiol levels are inversely associated with cortical porosity in older men. J Clin Endocrinol Metab. 2014;99(7):E1322-E1326.

26. Argoud T, Boutroy S, Claustrat B, Chapurlat $\mathrm{R}$, Szulc P. Association between sex steroid levels and bone microarchitecture in men: the STRAMBO study. JClin Endocrinol Metab. 2014;99(4):1400-1410.

27. Khosla S, et al. Relationship of volumetric BMD and structural parameters at different skeletal sites to sex steroid levels in men. J Bone Miner Res. 2005;20(5):730-740.

28. Khosla S. New insights into androgen and estrogen receptor regulation of the male skeleton. J Bone Miner Res. 2015;30(7):1134-1137.

29. Manolagas SC, O'Brien CA, Almeida M. The role of estrogen and androgen receptors in bone health and disease. Nat Rev Endocrinol. 2013;9(12):699-712.

30. Khosla S, Melton LJ 3rd, Riggs BL. The unitary model for estrogen deficiency and the pathogenesis of osteoporosis: is a revision needed? J Bone Miner Res. 2011;26(3):441-451.

31. Vandenput L, et al. High serum SHBG predicts incident vertebral fractures in elderly men [published online ahead of print September 22, 2015]. J Bone Miner Res. doi: 10.1002/jbmr.2718.

32. Vanderschueren D, et al. Sex steroid actions in 
male bone. Endocr Rev. 2014;35(6):906-960.

33. Ohlsson C, et al. Comparisons of immunoassay and mass spectrometry measurements of serum estradiol levels and their influence on clinical association studies in men. JClin Endocrinol Metab. 2013;98(6):E1097-E1102.

34. Doran PM, Riggs BL, Atkinson EJ, Khosla S. Effects of raloxifene, a selective estrogen receptor modulator, on bone turnover markers and serum sex steroid and lipid levels in elderly men.
J Bone Miner Res. 2001;16(11):2118-2125. 35. Smith MR, Malkowicz SB, Brawer MK, Hancock ML, Morton RA, Steiner MS. Toremifene decreases vertebral fractures in men younger than 80 years receiving androgen deprivation therapy for prostate cancer. J Urol. 2011;186(6):2239-2244.

36. Cauley JA, et al. Recruitment and screening for the testosterone trials. J Gerontol A Biol Sci Med Sci. 2015;70(9):1105-1111.
37. Cawthon PS, et al. Sex hormones and radiographic vertebral fractures in older men. J Bone Miner Res. 2014;28(suppl 1):SA0330.

38. Ettinger B, et al. Performance of FRAX in a cohort of community-dwelling, ambulatory older men: the Osteoporotic Fractures in Men (MrOS) study. Osteoporos Int. 2013;24(4):1185-1193.

39. Byberg L, et al. Prediction of fracture risk in men: a cohort study. J Bone Miner Res. 2012;27(4):797-807. 